\title{
Análisis de la capa de fibras nerviosas usando el GDx en pacientes pseudofáquicos con opacificación capsular posterior
}

\author{
Analysis of the nerve fiber layer using GDX in pseudophakic patients \\ with posterior capsular opacification
}

Apreciado Editor:

Hemos leído con interés el artículo de Arraes et al., publicado en el número de Enero/Febrero de 2008 en Arquivos Brasileiros de Oftalmología ${ }^{(1)}$. Los autores llevaron a cabo una investigación para valorar la influencia de la opacificación capsular posterior (OCP) sobre las estimaciones de la polarimetría láser (PL), realizando las mediciones de la PL mediante GDx antes y después de la capsulotomía Nd:YAG.

La PL es una técnica que estima el grosor de la capa de fibras nerviosas peripapilares de la retina (CFNPR). En el glaucoma la evaluación de la CFNPR es importante pues un adelgazamiento de la misma es uno de los signos más tempranos de esta enfermedad $^{(2)}$ que potencialmente es causante de ceguera. Teniendo en cuenta que la pseudofaquia en el glaucoma es un estado con alta prevalencia el tema estudiado es clínicamente importante.

Arraes et al. no encontraron diferencias significativas entre los parámetros de la PL antes y después de la capsulotomía $^{(1)}$.

Hace poco tiempo nuestro grupo publicó una serie de casos preliminar (incluyendo mapas de GDx y fotografías de las opacidades capsulares) seguido de un estudio clínico sobre el mismo tema ${ }^{(3-4)}$. Sin embargo nosotros concluimos que la OCP está asociada con cambios significativos en la PL. En resumen, nuestros resultados sugieren que las mediciones con PL pueden sobreestimar los parámetros de la CFNPR en ojos afectos de OCP.

Los métodos usados por Arraes et al., y nuestro grupo fueron similares. Sin embargo quedan algunas cuestiones pendientes que pueden hacer entender las diferencias en los resultados.

Como es sabido la birrefringencia del segmento anterior (BSA) tiene que ser compensada para asegurar una estimación adecuada de la CFNPR. En 1993 se introdujo un primer compensador de la BSA en el GDx que usaba una compensación corneal fija (FCC) basada en los valores medios poblacionales del eje y la magnitud de polarización. Sin embargo, debido a la alta variabilidad interindividual e intraindividual de la BSA, el uso de la CCF ofrecía en muchos casos mediciones erróneas de la CFNPR $^{(5)}$.

Posteriormente en 2002 se introdujo un compensador corneal variable (VCC) que permitió una compensación espe- cífica de la BSA de cada ojo, que ha permitido una buena discriminación entre ojos sanos y glaucomatosos ${ }^{(6)}$.

Los autores del referido estudio ${ }^{(1)}$ realizaron las exploraciones con GDx pero no mencionan si realizaron las compensaciones de la BSA mediante FCC o VCC. En caso de haberlas realizado mediante CCV no sabemos si se realizaron únicamente antes de la capsulotomía o si se realizaron antes y también después. Nuestros estudios fueron realizados con GDx-VCC y compensación de la BSA tanto antes como después de la eliminación de la OCP.

A propósito de la relación de la OCP y la BSA nuestro grupo ha publicado recientemente un estudio en una nueva serie de ojos afectos de OCP donde hemos observado que tanto el eje como la magnitud de polarización medidos con GDx-VCC cambian significativamente tras la capsulotomía así que consideramos aconsejable recompensar la BSA tras la eliminación de la $\mathrm{OCP}^{(7)}$. Además, los resultados obtenidos en dicho estudio reproducen nuevamente una sobreestimación de los parámetros de espesor de la CFNPR antes de la capsulotomía $^{(7)}$.

Por otra parte nos preguntamos si Arraes et al. valoraron la distribución normal de parámetros tales como el "Nerve Fiber Indicador" también conocido como "el número" ${ }^{(1)}$.

Los autores seguramente podrán aclarar estas cuestiones.

Palabras Clave: Lasers; Catarata; Extracción de catarata; Terapia por láser; Fibras nerviosas; Células ganglionares de la retina.

Keywords: Lasers; Cataract; Cataract extraction; Laser therapy; Nerve fibers; Retinal ganglion cells.

\section{REFERENCIAS}

1. Arraes TA, Cavalcanti HD, Arraes J, Leão AC, Sena MF. Análise da camada de fibras nervosas pelo GDx em pacientes pseudofácicos com opacidades da cápsula posterior. Arq Bras Oftalmol. 2008;71(1):75-8.

2. Sommer A, Katz J, Quigley HA, Miller NR, Robin AL, Richter RC, et al. Clinically detectable nerve fiber atrophy precedes the onset of glaucomatous field loss. Arch Ophthalmol. 1991;109(1):77-83.

3. García Medina JJ, García Medina M, Shahin M, Pinazo Durán MD. Posterior 
capsular opacification effects scanning laser polarimetry examination. Graefes Arch Clin Exp Ophthalmol. 2006;244(4):520-3.

4. García-Medina JJ, García-Medina M, Dorta SG, Pinazo-Durán MD, GallegoPinazo R, Zanón-Moreno VC. Effect of posterior capsular opacification removal on scanning laser polarimetry measurements. Graefes Arch Clin Exp Ophthalmol. 2006;244(11):1398-405.

5. Reus NJ, Colen TP, Lemij HG. Visualization of localized retinal nerve fiber layer defects with the GDx with individualized and with fixed compensation of anterior segment birefringence. Ophthalmology. 2003;110(8):1512-6.

6. Reus NJ, Lemij HG. Diagnostic accuracy of the GDx VCC for glaucoma.1. Ophthalmology. 2004;111(10):1860-5.

7. Garcia-Medina JJ, Garcia-Medina M, Zanon-Moreno VC, Gallego-Pinazo R, Pons-Vazquez S, Gonzalez-Ocampo S, et al. The influence of posterior capsular opacification removal on anterior segment birefringence parameters as measured by scanning laser polarimetry. Clin Experiment Ophthalmol. 2007; 35(5):414-20. Comment in: Clin Experiment Ophthalmol. 2007;35(5):401-2.
José Javier Garcia-Medina ${ }^{1}$ Manuel Garcia-Medina² María Dolores Pinazo-Duran ${ }^{3}$

${ }^{1}$ Doctor en Oftalmología por la Universidad de Valencia. Médico Oftalmólogo en el Hospital La Inmaculada de Huercal-Overa - Almería - España.

${ }^{2}$ Doctor en Oftalmología por la Universidad de Cádiz. Médico Oftalmólogo en el Complejo Hospitalario Torrecárdenas - Almería - España.

Doctor en Oftalmología por la Universidad de Valencia. Médico Oftalmólogo. Jefe de la Unidad de Investigación Oftalmológica "Santiago Grisolía" - Hospital Universitario Doctor Peset - Valencia - España.

Correspondencia: José Javier Garcia-Medina, MD, PhD. Hospital La Inmaculada, SAS. Avenida Dra. Ana Parra s/nº - 04600 Huercal-Overa - Almería - España. Teléfono: +34950029000

email: josegarciam@yahoo.com 